\title{
Chronic Disease Tool to Find Risk using Naïve Bayes Algorithm
}

\section{V.Khanaa}

\begin{abstract}
Significant for better infection the overseers, propelled interventions, and authentically effective valuable gatherings help dispersing. Exceptional AI approaches have been connected bits of knowledge in cutting edge prosperity document for this task. A variety of past undertakings, at any rate, based on formed fields and loses the incredible bit of assurances intimate the unstructured notes. On this artworks we exhort an exquisite play out phenomenal undertakings framework for perplexity beginning decision that links each free substance medicinal notes and took care of estimations. We view execution of balanced critical acing systems nearby LSTM, CNN and interesting leveled designs. Rather than standard substance based absolutely decision shape, our device does never again need misery unequivocal factor creating, and may manage nullifications and numerical qualities that exist inside the material. Our outcomes on an amigo of around 1 million sufferers show off that models the utilization of material defeat models utilizing basically made estimations, and that structures organize for the use of numerical traits and refutations in the substance, independent of the troublesome material, nearly advance performance. But, we watch changed commonness procedures for valuable pros to get to the base of variant estimates.
\end{abstract}

\section{INTRODUCTION}

The previous years has seen a taking off expansion of data in EHR structures. Dealt with understanding data, for example, financial aspects, lab results, malady history, systems and prescriptions, and unstructured data, for example, advance notes and release notes are gathered during each clinical experience. This makes a chance to mine the data to develop thought. In any case experts have bound time to procedure all the open information for every patient, let alone to see designs diagonally over for all intents and purposes indistinguishable patients. Man-made insight draws near, then again, are fitting for detaching data from giant extent of information and summarizing to new cases. Late assessments have demonstrated promising outcomes utilizing EHR and huge learning models to foresee clinical occasions. Regardless, past assessments overwhelmingly dependent on

displaying with dealt with information, for example, lab results, clinical estimations [1-2]. [3] predicted clinical mediation joining dealt with information and notes. At any rate the creator set out to changing every clinical story note to a 50-D vector of point degrees with LDA. These techniques

Revised Manuscript Received on October 22, 2019.

Dr. V. Khanaa, Professor, Information Technology, Bharath Institute of Higher Education and Research, Chennai, Tamilnadu, India. Drvkannan62@gmail.com

\section{Keywords: CNN, LSTM, AI, Chronic disease.}

need cutoff of detaching rich data from unstructured healing notes information. For instance, how a patient is picked up by their family members isn't coded in any case can be a pointer of a shocking thriving correspondingly as a present social help for the patient. A later report [4], endeavored to see ICD code task dependent on MIMIC release notes and indicated that critical learning based frameworks beats shallower ones.

In this assessment we propose a general structure for foreseeing beginning of infections that underline the going with: (1) Flexibility to use both substance and unstructured dealt with numerical attributes. Vector portrayal of words or course of action of substance, for example, pre-masterminded embeddings, can be feasibly joined with other mathematical information. (2) Multi-task structure that can be summarized to various sicknesses. We test model execution on the craving for three illness locales, to be unequivocal congestive heart bafflement, kidney disappointment and stroke. Since we do not need infection express part building or model structure, a practically identical arrangement can be immediately used to other ailment areas. (3) Evaluation on an enormous accomplice of authentic patient information, with different note types and note lengths.

We endeavor different things with assortments of noteworthy learning model structures, melding sporadic neural system with Convolutional Neural Networks (CNN) and Long Short-term Memory units (LSTM). Moreover we suggest a new framework for managing invalidations in this measure responsibility.[2]

To the degree implementation, we show that models utilizing helpful notes beat those with just lab and estimation information. Moreover, huge learning frameworks accomplish favored execution over essential apostatize plan with TF-IDF highlights. We locate that couple of models, especially a BiLSTM with invalidation imprints and lab and estimation highlights, can accomplish high AUC for all of the three diseases. [3]

So as to assist restorative authorities with interpreting model yield, we study the common sense of two or three depiction procedures to perceive the words and enunciations with unassumingly high effect on the model check. We discover log-Chances based system gives continuously ordinary depiction while slant based methodology will when in doubt be nonsensically clamorous. We further consider the sufficiency of two or three perception frameworks to perceive the explanations and words with sufficiently high effect on the model gauge. For instance, how a patient is gotten by their family members are not coded at any rate can be a marker of a repulsive thriving comparably as a present social help. These methods need most distant purpose of confining rich data from unstructured restorative 
notes information. To check the chronic illness.

\section{FORECAST STRUCTURE}

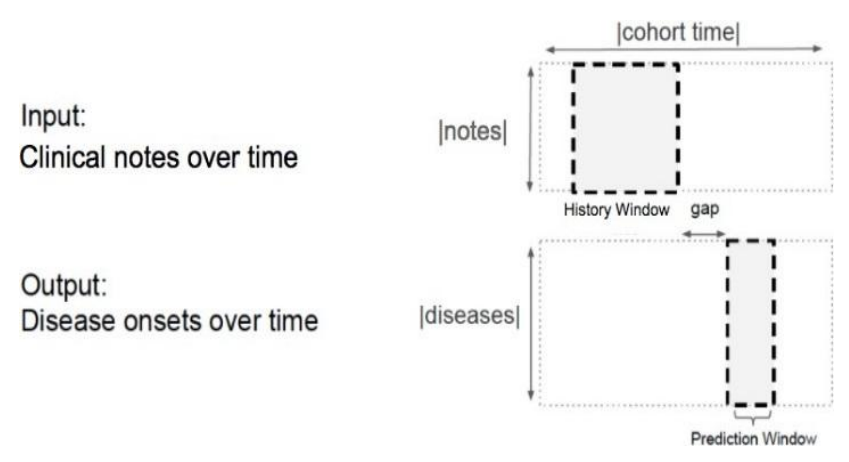

Figure 1: Diagram of forecast structure

Figure 1 represents the forecast structure. The objective is to estimate the beginning of diseases before finding time. We use data gathered in the history window to anticipate analyze in the forecast window. We likewise require a whole period between the finish of the history window and the start of the expectation window. The intention is to keep the model from swindling with data created directly before the conclusion time. In particular, we utilize a year verifiable window, a 3-month hole and a 6-month expectation window in this investigation.

\section{Associate}

We utilize restorative notes, socioeconomics and analyze in ICD-10 codes from the NYU Langone Hospital EHR framework. The information contains clinical experiences of more than 1 million patients somewhere in the range of 2014 and 2017, and in excess of 15 million passages of therapeutic notes. Figure8and Figure9in the Appendix demonstrates the dissemination of note types and note lengths by sort. Note lengths commonly run from hundreds to thousands of words, however a few notes are amazingly long with more than $10 \mathrm{~K}$ words. Segment 3.3.1provides subtleties on content preprocessing. [4]

Moreover, over $60 \%$ of experiences contain numerical data including indispensable signs and lab test esteems in the notes. We separate the most widely recognized ones from the content and use them as extra contribution to the model.

\section{LITERATURE SURVEY}

The objective of our assessment was to take a gander at various existing ceaseless infection models, their parts and their activity in the organization of Diabetes, Cardiovascular Disease (CVD) and Chronic Obstructive Pulmonary Disease (COPD). A composing search was performed using Pub Med and CINHAL during a period. Following key terms were used either in single or in mix, for instance, "Diabetes Mellitus" AND "Chronic Disease Model" OR "CVD" OR "COPD". An entirety of 23 investigations was consolidated into the last assessment. Predominant piece of the assessments were US-based. Five interminable malady models included Improving Chronic Illness Care (ICIC), Chronic Care Model (CCM), and Stanford Model (SM), Community based Transition Model (CBTM), and Innovative Care for Chronic Conditions (ICCC). CCM was the most thought model. Parts concentrated included movement system structure and clinical information system and decision help (57\%), self-organization support $(87 \%)$ and prosperity system affiliation (52\%). Segments including center consideration on the patient and understanding wellbeing (4\%), network arrangements (4\%), assembled coordinated social insurance (4\%), remote patient checking (4\%) and family (13\%) have not been very much considered. Different components including bolster change in perspective, oversee political condition, adjust sect oral strategies for wellbeing, use human services faculty all the more viably, bolster patients in their networks, stress anticipation, recognize tolerant explicit concerns identified with the progress procedure, and wellbeing education among visits and medications have likewise not been very much concentrated in the current writing. It was hazy to what degree the outcomes produced is pertinent to various populaces and areas and along these lines is a zone of future research. Future investigations are additionally expected to test chronic disease models in states where all the more ethnically and racially delegate patients get chronic consideration. Future to improvement ought to likewise incorporate data on different boundaries including transportation issues, funds and absence of administrations.[5]

Nowadays Kidney Disease is a developing issue in the around the world. Because of the high probability of death inside a brief timeframe, a patient must be hospitalized and suitably relieved. Numerous Data Mining strategies are utilized in the human services industry for anticipating the Kidney Disease. The Data Mining systems, to be specific SVM, Naive Bayes, Decision Tree, [6] Classification, Neural Network are utilized to break down the precision for the kidney related disease.

Chronic Kidney Disease (CKD) is a noteworthy therapeutic issue and can be restored whenever treated it in the beginning periods. Ordinarily, individuals don't know that medicinal tests we take for various purposes could contain important data concerning kidney diseases. Therefore, traits of different restorative tests are examined to recognize which qualities may contain supportive data about the disease. The goal of this paper is to utilize such properties. The data says that it causes us to gauge the seriousness of the issue, the anticipated survival of the patient after the sickness, the example of the disease and work for restoring the disease. Henceforth we considered an informational collection with various properties that can be found when all is said in done medicinal tests, AI is connected by building up a choice tree utilizing the $\mathrm{C} 4.5$ calculation and anticipated whether the individual is ordinary or experiencing kidney issue. This proposed model will be created utilizing Java language and is actualized in Net-Beans stage. [7]

\section{Target Disease Definition}

We describe the mapping among the ICD10 codes and the 3 target diseases with the guide of an alliance of offices for Medicare and Medicaid administrations ceaseless conditions and the Healthcare expense and use adventure logical Classifications programming, with help from restorative masters from NYU logical personnel..

\section{Modeling Dataset}

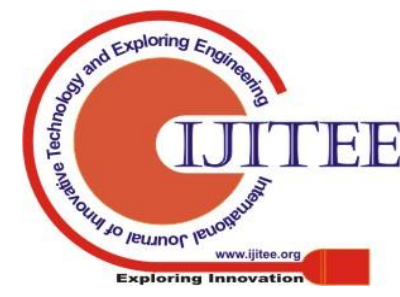


We restriction the examination to sufferers with in any event encounters within the chronicled window and at any price two encounters in the preference window. We further require the hole between the first and remaining encounters within the preference window to be longer than or proportionate to two months, to preserve up a key separation from the case of patient buying and selling centers and getting examined elsewhere. Additionally, patients with the target warranty in any encounters going earlier than the preference window are rejected, to prevent displaying sufferers who starting at now have the goal ailment. We make use of a hiding technique to manipulate disregard patients which might be big fashions for one sickness yet can be undermined for some other. As an example, an affected person might also beginning at now have heart frustration yet we have to want to expect the start of kidney disillusionment or stroke. The showing associate as wishes be diminishes to approximately $300 \mathrm{k}$ sufferers.

For patients inside the showing companion, we watch their encounters up to 3 years. Because the hard and rapid duration of the records window, the outlet term and the figure window is underneath 2 years, we use a three-month sliding window to utilize most of the facts. Hence, a comparative patient may also contribute various statistics centers. We by means of then cut up the showing records into getting equipped, endorsement and take a look at set by means of an volume of $7: 1: 2$ by patient identity. A comparable patient never seems in two separate sets.

Table 1: No. of Records by Target Disease ( Negative Cases: Positive Cases)

\begin{tabular}{lccc}
\hline Target & Training Set & Validation Set & Test Set \\
\hline Congestive HeartFailure & $644 \mathrm{~K}: 4080$ & $93 \mathrm{~K}: 574$ & $184 \mathrm{~K}: 1167$ \\
Kidney Failure & $616 \mathrm{~K}: 10051$ & $88 \mathrm{~K}: 1428$ & $176 \mathrm{~K}: 2809$ \\
Stroke & $653 \mathrm{~K}: 3195$ & $94 \mathrm{~K}: 406$ & $187 \mathrm{~K}: 916$ \\
\hline
\end{tabular}

\section{NAIVEBAYES ALGORITHMALGORITHM}

We utilize sorted out records on sufferers ethnicity, race, intercourse, and age from the EHR structure as measurement features had been shown to be impressive for ailment gauge. Ethnicity, race, and sexual direction are addressed as one-warm encoded straight out features. Age is addressed as a constant thing in years. This yields an aggregate of 61 more noteworthy features. Feature scatterings are given. Measurement features are accessible for about $90 \%$ of sufferers.

Lassstatic Dataset(Dataset):

\# Each record is: [[text_enc1, text_enc2, ...], [num_enc1, num_enc2, ...], disease, mask, age, gender, race, eth]

\footnotetext{
def init (self, root dir, dsName, nClassGender, nClassRace, nClassEthnic, transform=None):

self.root_dir=root_dir

self.ds $=$ json.load $($ open $($ root_dir + dsName, 'r') $)$

print('Loaded: ', dsName)
}

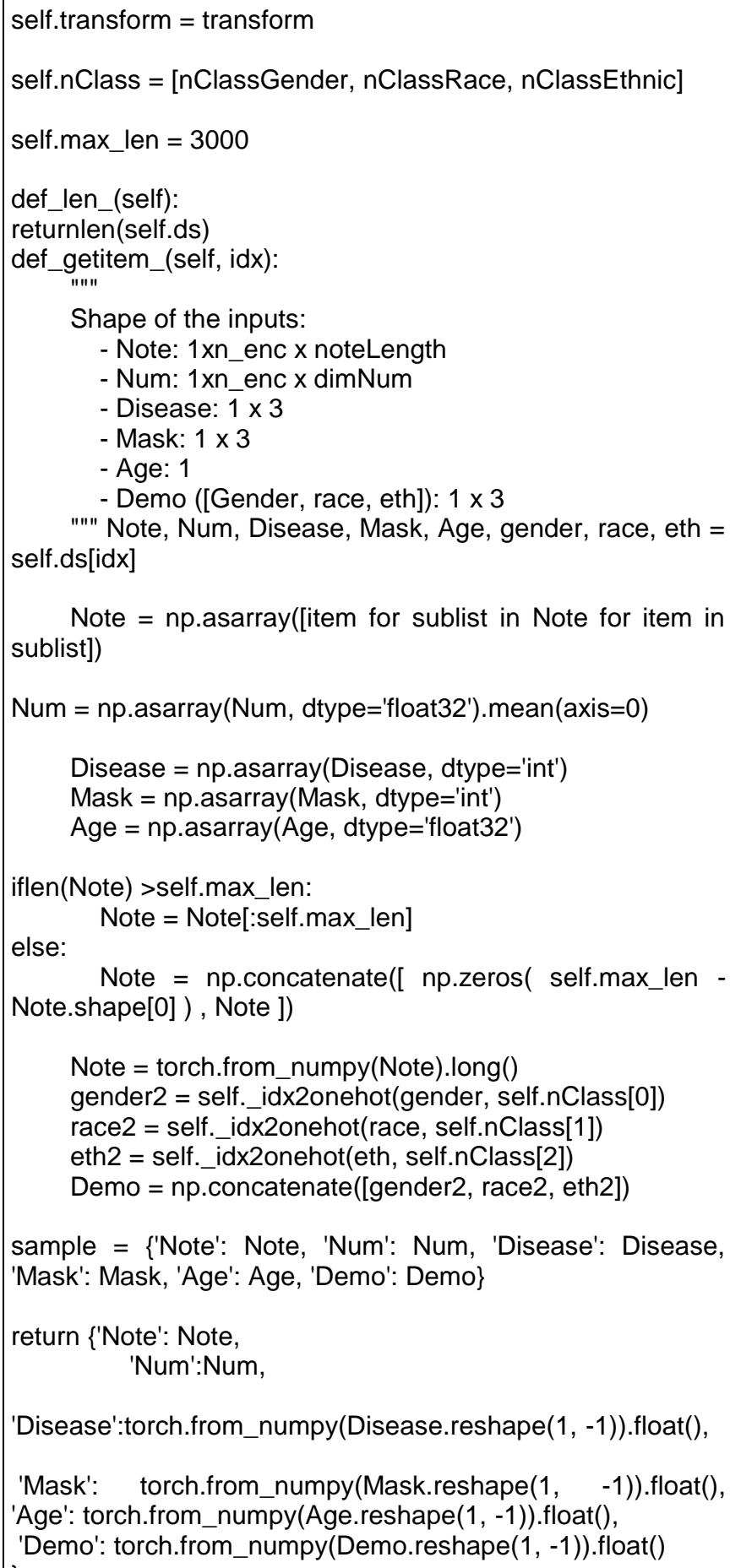

"'" Note, Num, Disease, Mask, Age, gender, race, eth $=$ sublist])

Note $=$ np.asarray $([$ item for sublist in Note for item in Num $=$ np.asarray $($ Num, dtype $=$ 'float32' $) \cdot$ mean $($ axis $=0)$

Disease $=$ np.asarray (Disease, dtype $=$ 'int')

Mask $=$ np.asarray (Mask, dtype $=$ 'int')

Age $=$ np.asarray $($ Age, dtype $=$ 'float32')

iflen(Note) >self.max_len:

Note $=$ Note[:self.max len]

else:

Note $=$ np.concatenate $([\mathrm{np} . z e r o s$ ( self.max_len Note.shape[0] ), Note ])

Note $=$ torch.from_numpy(Note).long()

gender2 $=$ self._idx2onehot(gender, self.nClass[0])

race2 $=$ self. idx2onehot $($ race, self.nClass[1])

eth2 $=$ self._idx2onehot(eth, self.nClass[2])

Demo $=$ np.concatenate $([g e n d e r 2$, race2, eth2] $)$

sample $=\{$ 'Note': Note, 'Num': Num, 'Disease': Disease, 'Mask': Mask, 'Age': Age, 'Demo': Demo\}

return \{'Note': Note,

'Num':Num,

'Disease':torch.from_numpy(Disease.reshape(1, -1)).float(),

'Mask': torch.from_numpy(Mask.reshape(1, -1)).float(), 'Age': torch.from_numpy(Age.reshape(1, -1)).float(), 'Demo': torch.from_numpy(Demo.reshape(1, -1)).float()

\section{DEEP LEARNING MODELS}




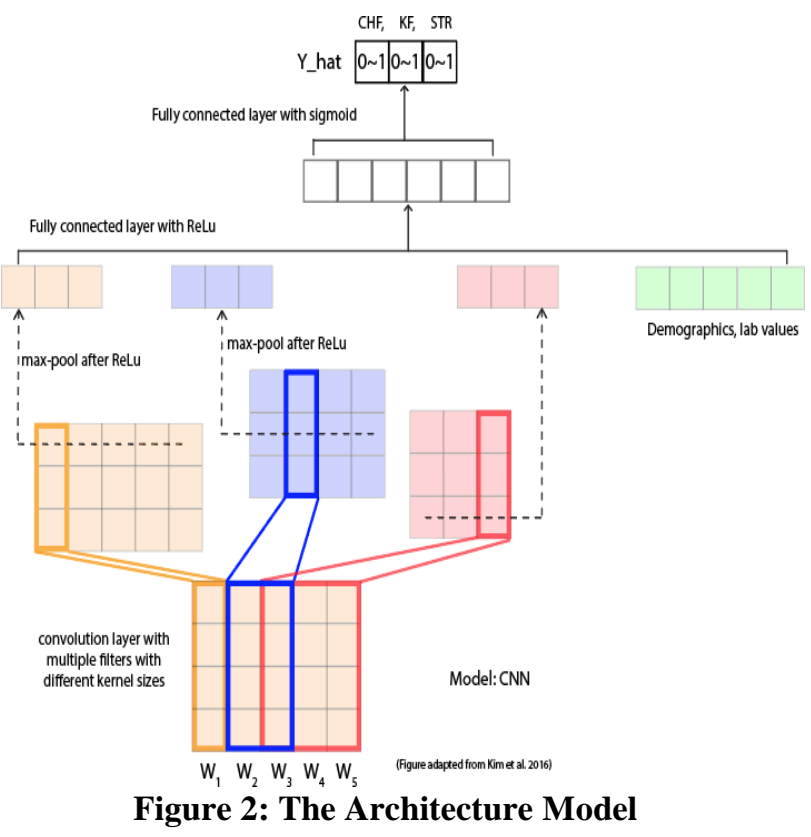

VI. CONVOLUTIONAL NEURAL NETWORK (CNN)

The Convolution Neural Network model relies upon the building in Kim. This model associates depictions of the substance at different degrees of reflection, fundamentally picks the most prominent n-grams from the notes. This depiction is supported through at any rate one totally related layers and a sigmoid yield layer to make a figure. We find that including a thick disguised layer after max-pooling incorporates judicious impetus as it can learn correspondences between the substance features. We interface all experience notes in the history time span all the way as commitment to this network. We join lab and measurement incorporates by interfacing the mean of every part inside the history window to the hid states after the most extreme pooling layer.

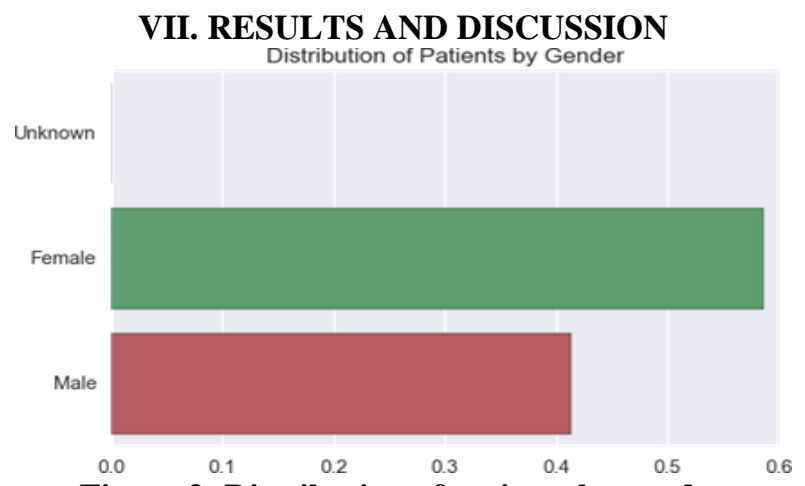

Figure 3: Distribution of patients by gender

The preference window to be longer than or proportionate to two months, to preserve up a key separation from the case of patient buying and selling centers and getting examined elsewhere. Additionally, patients by gender with the target warranty in any encounters going earlier than the preference window are rejected, to prevent displaying sufferers who starting at now have the goal ailment. We interface all experience notes in the history time span all the way as commitment to this network. We join lab and measurement incorporates by interfacing the mean. We make use of a hiding technique to manipulate disregard patients which might be big fashions for one sickness yet can be undermined for some other. As an example, an affected person might also beginning at now have heart frustration yet we have to want to expect the start of kidney disillusionment or stroke.

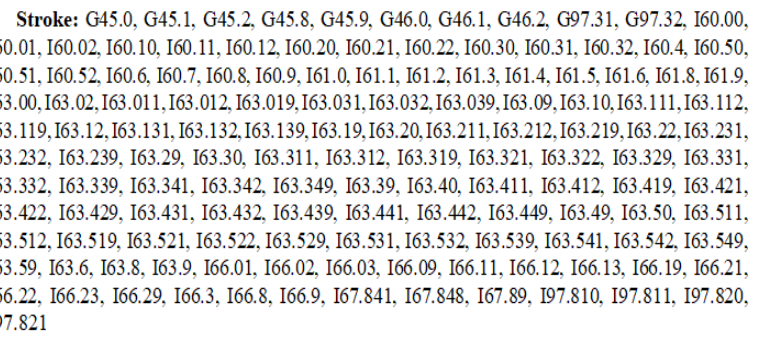

\section{Figure 2: Stroke Ranges}

\section{CONCLUSION}

The use of AI methods for sensible prosperity assessment works unprecedented. Since it empowers us to envision illnesses in earlier degrees, it spares the lives of individuals by technique for envisioning fixes. On this effort, we utilized the c4.5 thinking about figuring to predict subjects with never-ending kidney disappointment (ckd) illness and patients who do not (notckd) welcome the cleared out aftereffects of the malady. The desire for pollutions stays a significant therapeutic urges and test us to enlarge our endeavors to grow increasingly critical AI calculations to adroitly misuse estimations and concentrate the five star information.

Notwithstanding the manner in which that utilization of the relentless debasement models has been connected with sizeable updates in degrees of procedures of endless thought, it has never again normally been associated with progression in point of convergence of the street or entire arrangement outcomes. The most taking a gander at CCM segment to perform in fundamental thought has been clinical facts frameworks. Screwed up conceivable outcomes in cure the board.

Should clarify the clutch 22 of wandered forward framework execution without cutting edge affected individual effects. This will be settled through upgraded bits of knowledge and need help to the essential idea affiliation. Grouped all around based endeavors to advance development of protective associations have been made, yet a pair have insisted to be broadly appropriate or astounding. Colossal obstructions to progress are distinctive battling sales set upon essential thought workplaces and the very obliged extent of time accessible. To extend odds of social occasion and fulfillment, an involvement must be brief, facilitate into the development of patient visits, not build the time requests on authority time, and edify the affected individual provider conversation.

\section{REFERENCES}

1. B. Shneiderman. The eyes have it: a task by data type taxonomy for information visualizations. In Visual Languages, 1996. VL, IEEE, 336-343. September 1996.

2. E. Segel and J. Heer. Narrative visualization: Telling stories with data In Visualization and Computer Graphics 2010. IEEE Transactions, 16.6, 1139-1148. November-December 2010 . 
3. E. Tufte, E. The Visual Display of Quantitative Information. Graphics Pr, 2nd edition. May, 2001.

I. Bogost, S. Ferrari and B. Schweizer. Newsgames: Journalism at play. Cambridge, MA, MIT Press. 2010.

4. J. Beniger and D. Robyn. Quantitative graphics in Statistics: A brief history. In The American Statistician, vol. 32, no. 1, 1-11. 1978.

5. J. Harris (2011). Word clouds considered harmful. Nieman Journalism Lab. Retrieved from http://www.niemanlab.org/2011/10/word-clouds-considered-harmful/

6. J. Steele and N. Iliinsky. Beautiful Visualization: Looking at Data through the Eyes of Experts. Sebastipol, CA, O'Reilly. 2010.

7. M. Nichani and V. Rajamanickam. Interactive visual explainers - A simple classification. Elearning post 2003 url:http://www.elearningpost.com/articles/archives/interactive_visual _explainers_a_simple_classification.

8. S. Card and J. Mackinlay. The Structure of the Information Visualization Design Space Proceedings of IEEE Symposium on Information Visualization, IEEE Computer Society, 1997, 92.

9. T. Kapler and W. Wright. GeoTime Information Visualization. Information Visualization, 2004. INFOVIS 2004. INFVIS, IEEE, 25-32. October 2004.

10. U. Wiss and D. Carr. A Cognitive Classification Framework for 3Dimensional Information Visualization Luleå University of Technology, 1998.

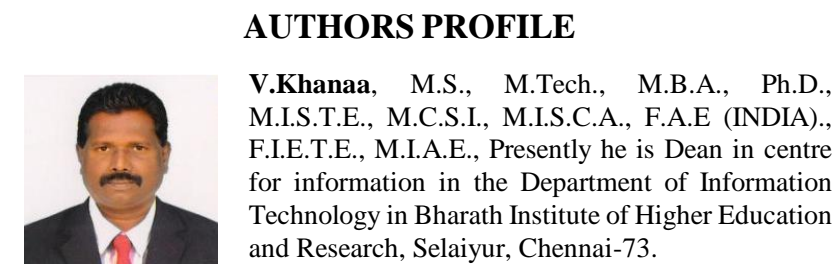

